\title{
Dynamical mean field study of the Mott transition in the half-filled Hubbard model on a triangular lattice
}

\author{
K. Aryanpour, ${ }^{1}$ W. E. Pickett,${ }^{1}$ and R. T. Scalettar ${ }^{1}$ \\ ${ }^{1}$ Department of Physics, University of California, Davis, California 95616
}

(Dated: April 13, 2018)

\begin{abstract}
We employ dynamical mean field theory (DMFT) with a Quantum Monte Carlo (QMC) atomic solver to investigate the finite temperature Mott transition in the Hubbard model with the nearest neighbor hopping on a triangular lattice at half-filling. We estimate the value of the critical interaction to be $U_{c}=12.0 \pm 0.5$ in units of the hopping amplitude $t$ through the evolution of the magnetic moment, spectral function, internal energy and specific heat as the interaction $U$ and temperature $T$ are varied. This work also presents a comparison between DMFT and finite size determinant Quantum Monte Carlo (DQMC) and a discussion of the advantages and limitations of both methods.
\end{abstract}

PACS numbers:

\section{MOTIVATION}

Systems with triangular lattice structure have been a source of attention mostly due to the frustration effects resulting from their non-bipartite structure. As a result of the competition between the frustration and strong electron correlations, these systems exhibit a wide range of exotic phases. Recent studies of the metalinsulator transition, superconductivity, and antiferromagnetism in the organic compounds $\kappa$-(BEDT-TTF $)_{2} \mathrm{X}$ with $\mathrm{X}$ as an anion ${ }^{1.2}$, discovery of superconductivity in $\mathrm{Na}_{x} \mathrm{CoO}_{2} \cdot \mathrm{yH}_{2} \mathrm{O}, \frac{3}{\underline{\underline{3}}}$ and the recent discovery of the Mott transitions in 0.33 monolayers of $\mathrm{Sn}$ on $\mathrm{Ge}(111)$ at 30 $\mathrm{K}, \stackrel{4}{=}$ as a few examples, underline the importance of these systems and their physics.

Theoretical work has been dedicated to the discovery of the magnetically ordered phases in the ground state of the Hubbard and $t-J$ models on triangular lattices as a function of the on-site electron-electron Coulomb inter-

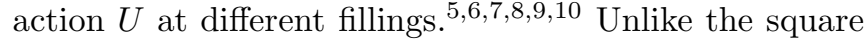
lattice at half-filling, which is a Mott insulator with antiferromagnetic order at arbitrarily small values of $U / t$, the ground state of a triangular lattice has a variety of magnetically ordered and disordered phases. This is due to the lack of perfect nesting in the non-interacting Fermi surface of a triangular lattice at half-filling.

For triangular lattices, according to the Hartree-Fock calculations of Krishnamurthy and co-workers,, 5.6 the Mott transition occurs from a paramagnetic metal to a paramagnetic insulator at half-filling for values of $U$ larger than the band width $W=9 t$. A variety of the physical properties of the triangular lattices including tendencies towards superconductivity within the small to intermediate $U$ regime have been studied at finite temperature using correlated electron approaches which go beyond mean field theory, such as the fluctuation exchange approximation (FLEX) $\stackrel{11}{\underline{1}}$ or one-loop renormalization-group 12 However, when $U / W \geq 1$, a more powerful cluster solving technique such as the Quantum Monte Carlo (QMC) is required to accurately describe the phase transition. Finite size lattice determi- nant Quantum Monte Carlo (DQMC) has already been employed for triangular and kagomé lattices ${ }^{13.14}$ Unfortunately, the finite size lattice QMC method incurs sign problems at both low temperatures and away from halffilling. In addition, due to the finite size nature of the problem, the non-local correlations are always overestimated and the system consisting of a finite size cluster becomes an insulator as soon as the correlation length reaches the size of the lattice at low enough $T$. Thus, the gaps or pseudogaps are also overestimated and are not guaranteed to persist as one reaches the thermodynamic limit 15 However, finite size effects can be reduced by size scaling and in general become less important as $U$ increases.

In this work, we employ the dynamical mean-field theory (DMFT) ${ }^{16.17 .18}$ to study the Mott transition for the Hubbard model on a triangular lattice at half-filling. DMFT describes systems in the thermodynamic limit and at the same time suppresses the physics due to the non-local correlations through coarse-graining over all momentum degrees of freedom. Therefore, susceptibilities can diverge, as in the thermodynamic limit but the Mott gap in the strong-coupling regime is always smaller than its counterpart given by a finite size lattice approach. The combination of the DMFT and QMC also does not incur sign problems at either low temperatures or any fillings which makes the low temperature physics more accessible and reliable.

DMFT is a more suitable approximation for the triangular lattice systems as opposed to those with square lattice structure. For instance, DMFT is incapable of describing the metal-insulator transition on the square lattice in the weak coupling regime. This is a consequence of the suppression of the short-range antiferromagnetic correlations responsible for this transition at small $U$ by the single site nature of DMFT $1 \frac{15}{1}$ However, in triangular lattices, due to the frustration of the lattice structure, even in the presence of non-local correlations, the short-range antiferromagnetic correlations are greatly suppressed. As a result, heavy quasiparticles form at the Fermi energy reminiscent of the DMFT physics $\frac{19}{19}$ 
DMFT has already been employed for studying triangular lattices using the exact diagonalization and Lanczos techniques ${ }^{20}$ In this paper, we present for the first time, the combination of DMFT and QMC for solving the Hubbard model at half-filling on a triangular lattice. By computing the density of states at different values of interaction $U$ and temperature $T$, we estimate the value of the critical interaction for the Mott transition to be $U_{c} / t=12.0 \pm 0.5$, less than the predicted value of 15 in Ref. 20, but, as expected, larger than the mean field result. In addition, the Mott transition features are also manifest in the evolution of the magnetic moment, total internal energy and the specific heat as functions of on-site interaction $U$ and temperature $T$.

\section{MODEL AND FORMALISM}

We consider the repulsive Hubbard model on a triangular lattice,

$$
\begin{aligned}
H= & -t \sum_{<i j>, \sigma}\left(c_{i \sigma}^{\dagger} c_{j \sigma}+c_{j \sigma}^{\dagger} c_{i \sigma}\right) \\
& -\mu \sum_{i \sigma} c_{i \sigma}^{\dagger} c_{i \sigma}+U \sum_{i} n_{i \uparrow} n_{i \downarrow},
\end{aligned}
$$

with $t$ the hopping amplitude, $\mu$ the chemical potential and $U$ the on-site Coulomb interaction between the electrons of opposite spins residing on lattice site $i$. Note that unlike the square lattice, on a triangular lattice, due to the lack of particle-hole symmetry, $\mu=U / 2$ does not yield half-filling. Also, this model exhibits quite different physics under the change of the sign of $t$, especially away from half-filling. The dispersion in the $U=0$ limit including the nearest neighbor hopping only is,

$$
\begin{array}{r}
\epsilon_{k}=-2 t\left[\cos \left(k_{x}\right)+\cos \left(\frac{k_{x}}{2}+\frac{\sqrt{3}}{2} k_{y}\right)+\right. \\
\left.\cos \left(\frac{k_{x}}{2}-\frac{\sqrt{3}}{2} k_{y}\right)\right] .
\end{array}
$$

The resulting noninteracting density of states is markedly asymmetric about $E=0$, extending from $E=-6 t$ to $E=+3 t$, with a van Hove singularity at $E=t$.

The DMFT approach to tight binding Hamiltonians starts by coarse-graining the Green's function,

$$
\begin{aligned}
& G_{\sigma}\left(i \omega_{n}\right)=\frac{1}{N} \sum_{k} G_{\sigma}\left(k, i \omega_{n}\right)= \\
& \frac{1}{N} \sum_{k} \frac{1}{i \omega_{n}-\epsilon_{k}+\mu-\Sigma_{\sigma}\left(i \omega_{n}\right)},
\end{aligned}
$$

where $\omega_{n}=(2 n+1) \pi T$ is the Matsubara fermionic frequency and $\Sigma_{\sigma}\left(i \omega_{n}\right)$ the local self-energy for spin $\sigma$. Note that the self-energy $\Sigma_{\sigma}\left(i \omega_{n}\right)$ in DMFT is local (momentum independent). In the first DMFT cycle iteration, we chose $\Sigma_{\sigma}\left(i \omega_{n}\right)=0$. The bath Green's function is then computed,

$$
\mathcal{G}_{\sigma}\left(i \omega_{n}\right)=\left(G_{\sigma}^{-1}\left(i \omega_{n}\right)+\Sigma_{\sigma}\left(i \omega_{n}\right)\right)^{-1} .
$$

$\mathcal{G}_{\sigma}\left(i \omega_{n}\right)$ is used as an input to the QMC solver. Our DMFT-QMC solver is based on the Hirsch-Fye algorithm ${ }^{21,22}$ in which the interacting two-body term in the Hubbard model is decomposed into two one-body density terms coupled to a Hubbard-Stratonovich field. Through the stochastic averaging over the most probable configurations of these Ising like fields, one calculates the fully interacting Green's function on a single site of a lattice $G_{\sigma}\left(i \omega_{n}\right)$. The local self-energy $\Sigma_{\sigma}\left(i \omega_{n}\right)$ is then calculated from the full and bath Green's functions $G_{\sigma}\left(i \omega_{n}\right)$ and $\mathcal{G}_{\sigma}\left(i \omega_{n}\right)$ respectively,

$$
\Sigma_{\sigma}\left(i \omega_{n}\right)=\mathcal{G}_{\sigma}^{-1}\left(i \omega_{n}\right)-G_{\sigma}^{-1}\left(i \omega_{n}\right) .
$$

This self-energy is again inserted in Eq 3 and the selfconsistent process continues until convergence.

The final product of the DMFT-QMC cycle is the full Green's function in imaginary time $G(\tau)$ which is used to compute the density of states (DOS) $N(\omega)$,

$$
G(\tau)=T \sum_{n} e^{-i \omega_{n} \tau} G\left(i \omega_{n}\right)=\int d \omega \frac{N(\omega)}{1+e^{-\beta \omega}} e^{-\omega \tau} .
$$

$N(\omega)$ must be calculated by inverting Eq 6 which is an ill-posed problem due to the statistical errors in $G(\tau)$ from the QMC simulations. This task in this paper is done by using the maximum entropy (MaxEnt) analytical continuation technique developed by Jarrell and Gubernatis ${ }^{23}$ This technique is based on Bayesian inference in which an entropy function with respect to an original default model is defined as a function of $N(\omega)$. The best $N(\omega)$ is the one that maximizes this entropy function for a given $G(\tau)$.

We also present results for the double occupation,

$$
<D>=<n_{i \uparrow} n_{i \downarrow}>
$$

which is suppressed by the on-site repulsion. The double occupation is related to the magnetic moment,

$$
\begin{gathered}
<m_{z i}^{2}>=<\left(n_{i \uparrow}-n_{i \downarrow}\right)^{2}>= \\
<n_{i \uparrow}>+<n_{i \downarrow}>-2<D>.
\end{gathered}
$$

The total internal energy can also be computed in this formalism in terms of the full Green's function and double occupation:

$$
E_{i n t}=\frac{T}{N} \sum_{n k \sigma}\left[\epsilon_{k} G_{\sigma}\left(k, i \omega_{n}\right)\right] e^{i \omega_{n} 0^{+}}+U D,
$$

with $D$ defined as in $\mathrm{Eq} 7 \mathrm{Eq} 9$ is equivalent to the Galitskii-Migdal expression for the total internal energy ${ }^{24}$ The specific heat at constant volume is

$$
C_{V}=\left(\frac{\partial E_{\text {int }}}{\partial T}\right)_{V}
$$


As seen later in this article, the variation of the total internal energy and specific heat as a function of $U$ and $T$ will exhibit features reflecting the Mott metal-insulator phase transition.

\section{RESULTS}

Before presenting our data for the density of states, we comment on some of the details of the maximum entropy procedure, since careful characterization of the data is essential to obtain reliable results. In this work, the DMFT-QMC cycle as described in section III converges at a desired tolerance which is about few percent for the time Green's function $G(\tau)$. The chemical potential is also adjusted simultaneously to keep the total occupation at half-filling. For the QMC runs, we adopt the criterion $U(\Delta \tau)^{2}<0.05$ with $\Delta \tau=\beta / L$ for finding $L$, the number of the time slices. $L$ can be small at high temperatures, however, even for temperatures as high as the bandwidth, we choose the lower limit of $L=40$. The number of the lattice sweeps for the QMC runs varies between $N=2 \times 10^{5}$ to $1 \times 10^{6}$ depending on the values of $U$ and $T$ and the reliability of the MaxEnt results. The outputs of the cycle are the fully interacting time Green's function, the magnetic moment on a single site and total internal energy for the temperature and interaction at which the QMC cycle has been running. The full $G(\tau)$ is employed to calculate the DOS through Eq[6] using the MaxEnt technique ${ }^{23}$

Care must be taken when using MaxEnt as the results can strongly depend on the quality of the QMC data. MaxEnt, as described in Ref. 23, requires statistically independent and Gaussianly distributed measurements of $G(\tau)$ for every time slice $\tau$ from QMC. Both these two properties are often lacking in the $G(\tau)$ data initially obtained from the QMC output because of the presence of correlations between the measurements. The solution is a careful rebinning of the $G(\tau)$ data which eliminates these correlations and yields Gaussianly distributed data. Correlations between different time slices are also eliminated in the MaxEnt code by diagonalizing a covariance matrix coupling different time slices together ${ }^{23}$

The other concern is a proper choice for the MaxEnt default model. In general, for good QMC data, the choice of the default model should not change the qualitative features of the spectral function significantly. Among the available default models, the Gaussian and second order perturbation models have been most commonly utilized. In our work, we adopt the latter and perform the so called annealing method in which we start with the second-order perturbation theory default model at a high temperature close to the band width. We then use the MaxEnt output for that temperature as the default model for a slightly lower temperature and continue until we arrive at the lowest desired $T$. Finally, as introduced in Ref. 23, there are different methods of doing the MaxEnt including the historic, classic and Bryan.

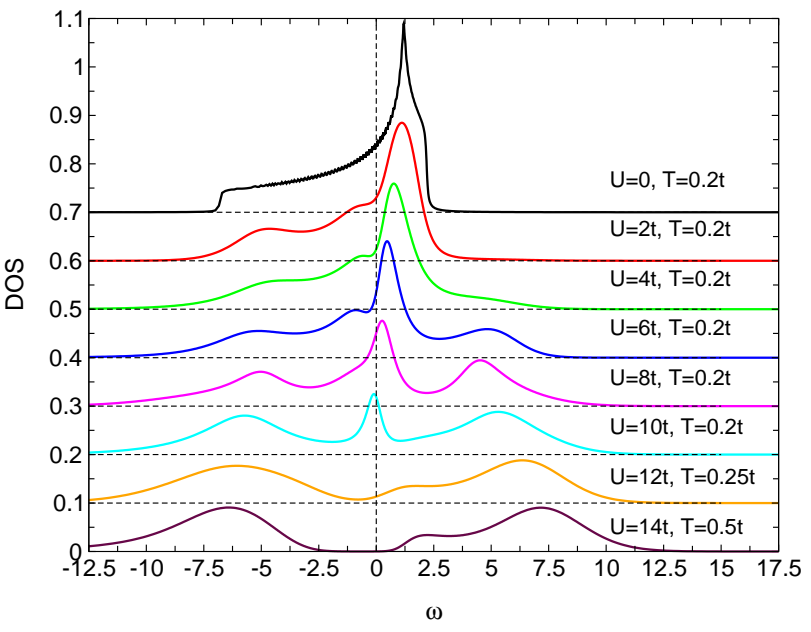

FIG. 1: (color on-line) The evolution of the density of states at half-filling for the triangular lattice as a function of $U$. For clarity, the base lines have been shifted in steps of 0.1 for different values of $U$. The $\omega=0$ line determines the location of the Fermi energy. Three behaviors are visible: At weak coupling the density of states has a finite value at $\omega=0$. At large coupling the density of states vanishes, signaling the opening of the Mott insulating gap. At intermediate coupling the density of states exhibits a Kondo resonance at $\omega=0$.

We utilized both classic and Bryan techniques in our calculations and found that both these two methods give qualitatively similar results.

In Fig 1 we present the evolution of the DOS as a function of $U$ at low temperatures. At $U=0$, the DOS shows a Van Hove singularity near $\omega=t$. As the interaction is gradually turned on, there is an overall broadening of the density of states, including a smearing out of the Van Hove singularity. The DOS at $U=8 t$ and $T=0.2 t$ exhibits the onset of the Abrikosov-Suhl (Kondo) resonance in which there appears a sharp quasi-particle peak near the Fermi energy with two shoulders, the upper and lower Hubbard bands, around it. For $U=10 t$ and $T=0.2 t$, the peak lines up with the Fermi energy and shoulders are approximately located at $\pm 5 t \approx \pm U / 2$. At $U=12 t$, and $T=0.25 t$, we clearly observe a different trend in which the Kondo resonance is absent and the DOS has a pronounced minimum at the Fermi surface, indicating that the system has been driven to the metal-insulator transition regime. For $U=14 t$, and $T=0.5 t$, the (Mott) gap is well established and the system becomes an insulator.

To better address the role played by temperature in the metal-insulator transition, in Fig 2 the DOS for three values of $U=10 t, 12 t$ and $14 t$ and different temperatures have been plotted. At $U=10 t$, as $T$ decreases, the DOS evolves towards a sharp Abrikosov-Suhl resonant quasi-particle peak. However, $U=12 t$ exhibits a completely different trend. As the temperature drops, the DOS evolves towards the opening of a gap. At $T=0.25 t$, we noticed that the MaxEnt results are of less reliability due to the correlations in the QMC data which could not be totally removed up to $10^{6} \mathrm{QMC}$ lattice sweeps by 

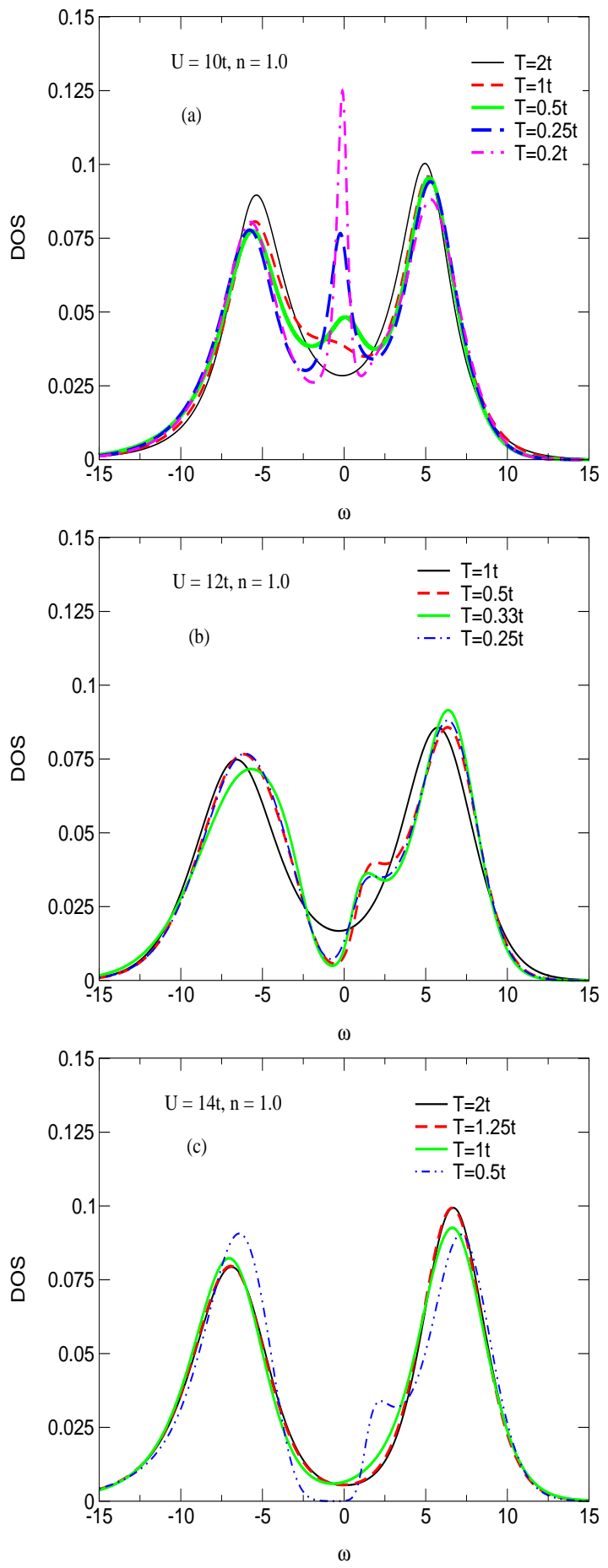

FIG. 2: (color on-line) Panel (a): the DOS for $U=10 t$ at half-filling and different temperatures. As the temperature is lowered a sharp Kondo resonance develops at $\omega=0$. The system is metallic. Panel (b): the same results as for panel (a) for $U=12 t$. The system is on the verge of insulating behavior. Panel (c): $U=14 t$. A Mott gap has opened at $T=0.5 t$.

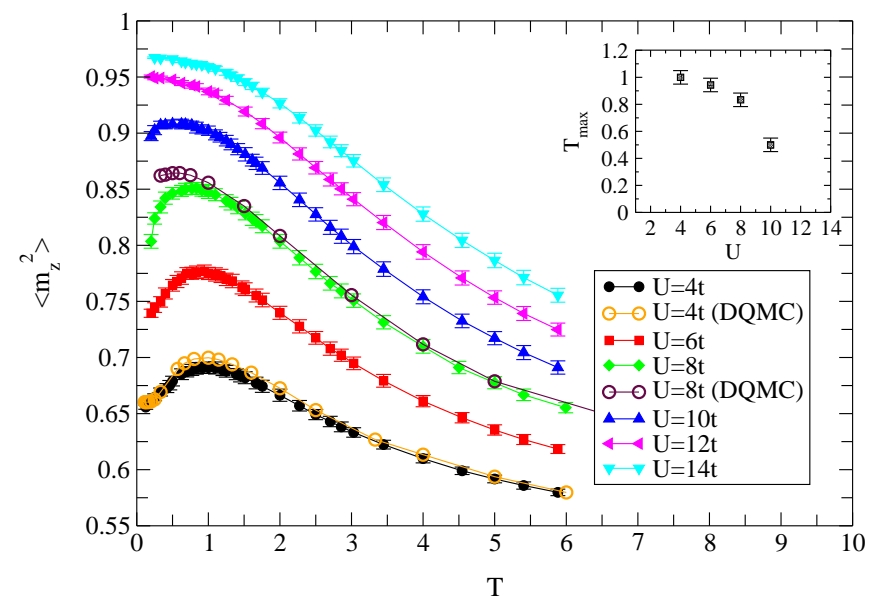

FIG. 3: (color on-line) Variation of the magnetic moment with temperature for different values of interaction $U$ at halffilling. $\left\langle m_{z}^{2}>\right.$ drops down close to its minimum 0.50 at large temperatures (cut off in this figure). The comparison between the DQMC and DMFT at $U=4 t$ and $8 t$ is also presented. The inset shows how $T_{\max }$, the temperature for the maximum $<m_{z}^{2}>$, drops as $U$ increases.

rebinning the data. (It is, of course, typical of Monte Carlo simulations that statistical fluctuations are largest near phase transitions) This is additional evidence that $U=12 t$ is close to the critical value for the opening of a gap but we were not able to determine conclusively whether the gap exists at $U=12 t$ if one can reach low enough temperatures. On the other hand, by the time $U=14 t$ (at $T=0.5 t$ ), the MaxEnt results show a pronounced Mott gap in the DOS. Therefore, it is clear that the critical value of $U_{c}$ must lie very close to $U=12 t$, leading to our estimate $U=12 t \pm 0.5 t$. This value is smaller than the value $U_{c} \approx 15 t$ obtained in Ref. 20 using the DMFT with exact diagonalization and Lanczos techniques as the impurity solvers, and comparable to $U_{c} \approx 12 t$ in Ref. 7 with exact diagonalization on 12 site lattices. At both values of $U=12 t$ and $14 t$, we also observe the appearance of a feature on the right hand side of $\omega=0$. This feature is likely a remnant of the peak in the non-interacting density of states- it disappears as $U$ is increased to larger values and is completely absent for $U=16 t$.

Another signature of the metal-insulator (Mott) transition is manifest in the behavior of the magnetic moment defined in $\mathrm{Eq}[\mathrm{l}$ as a function of temperature $T$ and interaction $U$. As shown in Fig 3 for small to intermediate values of $U,<m_{z}^{2}>$ reaches a maximum at a value of $T_{\max }$ which is characteristic of a Fermi-liquid dominated by spin fluctuations at low temperatures $15,26.27$ At low temperatures with an entropy of $\gamma T$ per particle, a Fermi liquid gains free energy upon heating by trying to localize the electrons in order to take advantage of a larger spin entropy ${ }^{26.27}$ The inset in Fig [3] shows how $T_{\max }$ decreases with $U$. However, at the onset of the Mott transition, $<m_{z}^{2}>$ becomes a monotonically decreasing function of 
$T$ as seen in Fig. 3 for $U=12 t$ and $14 t$. In a localized Mott phase, the spin entropy at $T \approx 0$ is finite and the system cannot gain free energy by further localizing the electrons upon heating. Comparison has also been made between DQMC on a $6 \times 6$ finite size lattice and DMFT in Fig 3 for values of $U=4 t$ and $8 t$. The agreement between the two approaches is very close at $U=4 t$ down to $T=0.125 t$. However, for $U=8 t$, at low temperatures, the DQMC approach exhibits a rather flat region for $\left\langle m_{z}^{2}>\right.$ below $T \approx 1 t$ in place of the downward curve in DMFT. This flat feature is similar to what is observed at larger values of $U$ in DMFT as a precursor to the insulating phase. This is typical of any finite size lattice approach such as DQMC in which at low enough temperatures, once the correlation length exceeds the size of the lattice, the system turns insulating. In the DMFT, on the other hand, the system is in the thermodynamic limit and therefore, the insulating phase features do not appear except at large enough interaction $U$ values. Nevertheless, DQMC has the advantage of including the non-local correlations which are ignored in DMFT. These correlations work in favor of the gap formation. Thus, DMFT overestimates the value of $U$ for the formation of the gap in the Mott transition.

The evolution of the total internal energy and specific heat defined in $\mathrm{Eq} 9$ and $\mathrm{Eq} 10$ respectively also exhibits signatures of a metal-insulator phase transition at large interaction $U$ values and low temperatures $T$. In Fig 4 panel (a), the total internal energy has been plotted as a function of temperature $T$ for different values of interaction $U$. We have employed a polynomial fitting procedure developed by Duffy and Moreo 28 and obtained the curves presented in panel (b). By analytically differentiating the polynomial fits with respect to temperature, we arrive at results for the specific heat plotted in panel (c). For values of $U=4 t$ to $10 t$, we observe a two-peak structure in the $C_{V}$ consistent with the results in Ref. 27.28 for the infinite dimensional and $2 D$ hypercubic Hubbard models respectively. The peak at low temperatures corresponds to the local spin fluctuations analogous to the formation of the Kondo singlets in the Kondo model. It is also a signature for the validity of the Fermi liquid picture as it indicates the existence of a conducting electron bath around the magnetic impurity $\underset{27}{\underline{2}}$ The upper peak at high temperatures, however, corresponds to on-site charge fluctuations. Separation between these two peaks occurs at $U=4 t$ and develops up to $U=8 t$ resulting in a sharp lower peak.

As prescribed in Ref. 28, the lower and higher temperature segments of $E_{\text {int }}(T)$ have been fit with two different polynomials which meet around $T \approx 1 t$. This prescription explains the rather sharp feature in the $C_{V}$ curve at $U=4 t$ in Fig 4 panel (c), around $T \approx 1.25 t$. However, the necessity of using two polynomials originates in the existence of two separate temperature regions, one dominated by spin and the other by charge fluctuations. The lower peak dwindles at $U=10 t$ as a sign of approaching the insulating phase ${ }^{29}$ It is also striking that, similar to the result of Ref. 27, within the Fermi liquid range up to $U=10 t$, all $C_{V}$ curves more or less intersect at the same temperature close to $T=2.1 t$. The picture however is different at $U=14 t$ when the insulating phase sets in. The lower peak completely vanishes and the $C_{V}$ curve goes to zero at a finite value of $T$. This is consistent with the behavior of $C_{V}$ in the presence of a gap $\Delta$ going as $e^{-\Delta / T}$ at low temperatures, and having a broad peak at high temperatures resulting from charge fluctuations in the upper Hubbard band ${ }^{27}$ For $U=12 t, E_{\text {int }}$ results were too noisy to be conclusive and therefore are not presented. This was apparently due to $U=12 t$ being close to the critical $U_{c}$ which made it difficult to achieve convergence in the simulation.

Lastly, Fig 5 presents a comparison between DMFT and DQMC for $E_{\text {int }}(T)$ at $U=4 t$ and $8 t$. Similar to the trend observed for $\left\langle m_{z}^{2}\right\rangle$ in Fig [3 at high enough $T$, the agreement betweeen the two approaches is clearly manifest in the results. For $U=4 t$ at low $T$, DQMC exhibits an insulating phase feature in the rather flat tail of the $E_{\text {int }}(t)$ curve near $T=0$, typical of a finite size approach as discussed earlier. For $U=8 t$, however, the effect of non-local correlations becomes more important and at the same time, finite size effects diminish. Hence, DQMC features may be more realistic in showing insulating phase signatures. Nevertheless, $U=8 t$ is still below the bandwidth $W=9 t$ and we do not expect to have entered the insulating phase as yet. Unfortunately, mostly due to the sign problem in DQMC, attempts in going to lower temperatures and higher $U$ values have not yet been successful. Thus, it is not clear to us which approach more plausibly describes the physics for the intermediate value of $U=8 t$.

In order to bring the DQMC and DMFT treatments into full agreement, it would be necessary to extrapolate both to a zero value of the discretization size of the inverse temperature $\beta$ and also to extrapolate DQMC and DMFT to infinite spatial cluster size and full momentum resolution (the dynamical cluster approximation (DCA)), respectively.

\section{CONCLUSIONS}

In summary, we have performed the first implementation of the DMFT using QMC as the solver for the halffilled triangular lattice Hubbard model. Our approach confirms the previous predictions that for large enough $U / W$, there is a metal-insulator (Mott) phase transition from a paramagnetic metal to a paramagnetic insulator at finite temperature. We demonstrate this phase transition by presenting the behavior of the DOS, magnetic moment on a single site, total internal energy and specific heat as functions of the interaction $U$ and temperature $T$. Our results suggest a critical value of interaction $U_{c} / t=12.0 \pm 0.5$. This is consistent with the value $U_{c} \approx 12 t$ from exact diagonalization on 12 site lattices 7 and $U_{c} \approx 15 t$ from DMFT with exact diagonalization 

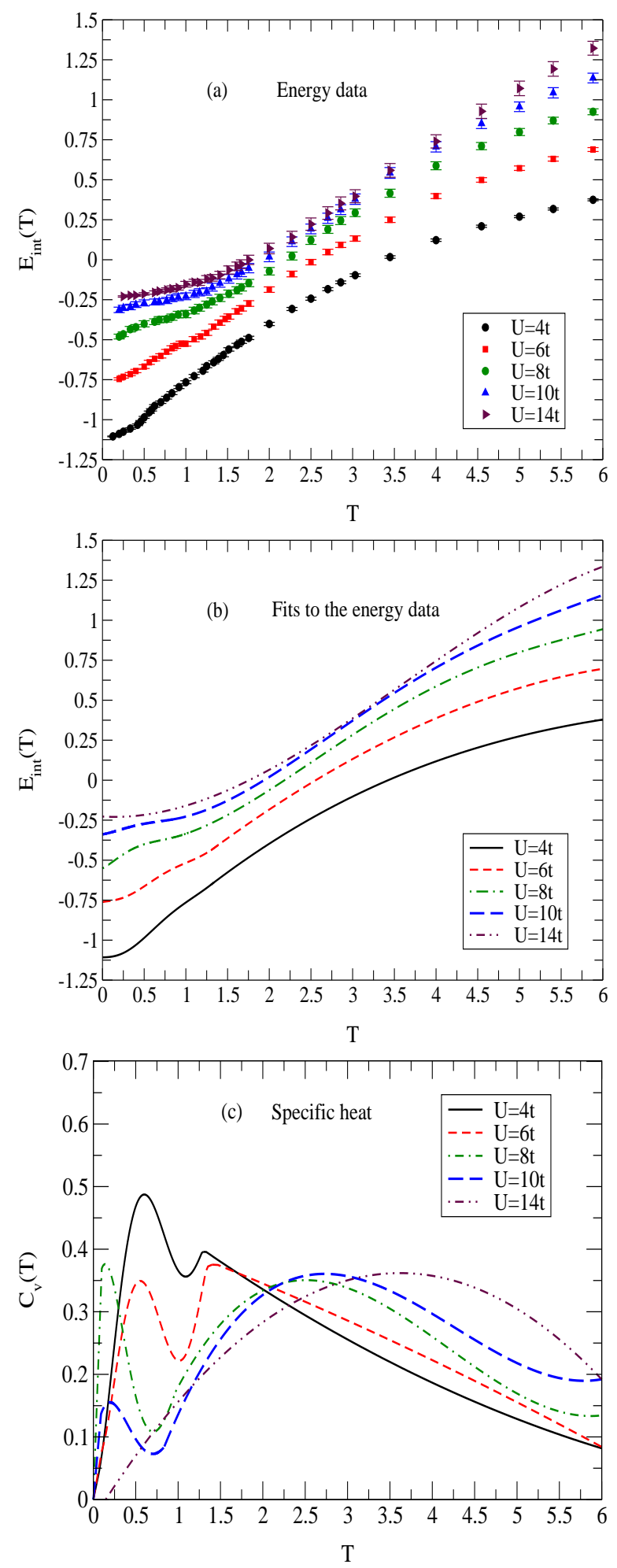

FIG. 4: (color on-line) Panel (a): Variation of the total internal energy versus temperature $T$ for different values of interaction $U$ including the error bars. Panel (b): Polynomial fits to data in panel (a). Panel (c): The specific heat as a function of temperature $T$ for different values of interaction $U$ taken by analytical differentiation of polynomials in panel (b).

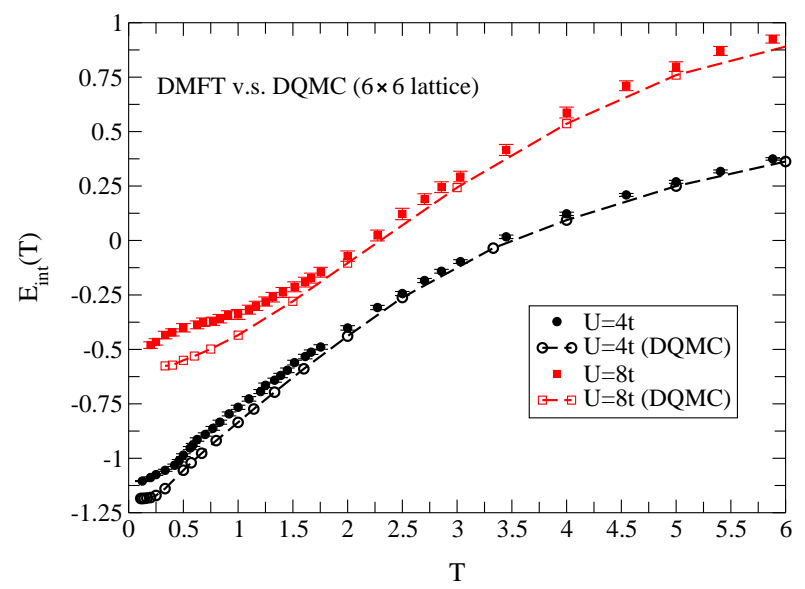

FIG. 5: (color on-line) Comparison between DMFT and DQMC on a $6 \times 6$ finite size lattice for the total internal energy versus temperature $T$ for interaction values of $U=4 t$ and $8 t$.

and Lanczos impurity solvers $\stackrel{20}{20}$. We have also investigated the intermediate interacting regime which is characterized by the presence of an Abrikosov-Suhl (Kondo) resonance.

DMFT is a more suitable approximation for studying the Mott transition on a triangular lattice compared to a square lattice. The reason is that in a triangular lattice, due to the frustration of the lattice structure, the shortrange antiferromagnetic correlations are suppressed and do not play a major role in the metal-insulator transition at small $U \underline{\underline{19}}$ Hence, neglecting these correlations, as done by DMFT, should still give us a qualitatively correct physical picture at least in the large $U$ limit. The combination of the DMFT and QMC also does not encounter the difficulties related to the sign problem at low temperatures and away from half-filling and also finite size effects associated with DQMC at low $T$. Thus, the low temperature physics is better described by the DMFT due to being in the thermodynamic limit. Nevertheless, DQMC has the advantage of incorporating non-local correlations which act in favor of gap formation and DMFT overestimates the value of $U_{c}$ because of lacking these correlations.

Due to the single site nature of the DMFT, we were not able to address the physics of magnetically ordered phases for triangular lattices as one approaches the $T=0$ limit. These studies have already been performed using the DQMC techniques ${ }^{13.14}$ However, the combination of the dynamical cluster approximation (DCA) and $\mathrm{QMC}^{30,31.32}$ can also be considered a promising future candidate for these studies as a complementary approach to DQMC. Work on different magnetically ordered phases at different coupling regimes, including ferromagnetism away from half-filling as discussed in Ref 20 is in progress. 


\section{ACKNOWLEDGMENTS}

We acknowledge useful conversations with M. Jarrell, A. Macridin, and S. Savrasov. This research was sup- ported by DOE DE-FG03-03NA00071. and DE-FG0106NA26204.
1 K. Kanoda, Physica C 282-287, 299 (1997); K. Kanoda, Hyperfine Interact. 104, 235 (1997).

2 R. H. McKenzie, Science, 278, 820 (1997).

3 K. Takada, H. Sakurai, E. Takayama-Muromachi, F. Izumi, R. A. Dilanian, and T. Sasaki, Nature (London) 422, 53 (2003).

${ }^{4}$ R. Cortés, A. Tejeda, J. Lobo, C. Didiot, B. Kierren, D. Malterre, E. G. Michel, and A. Mascaraque, cond-mat/0601029

5 H. R. Krishnamurthy, C. Jayaprakash, S. Sarker, and W. Wenzel, Phys. Rev. Lett. 64, 950 (1990).

${ }^{6}$ C. Jayaprakash, H. R. Krishnamurthy, S. Sarker, and W. Wenzel, Europhys. Lett. 15, 625 (1991).

7 M. Capone, L. Capriotti, F. Becca and S. Caprara, Phys. Rev. B 63, 085104 (2001).

8 H. Morita, S. Watanabe, and M. Imada, J. Phys. Soc. Jpn. $\mathbf{7 1}$ (2002) 2109

9 P. Srivastava and A. Singh, Phys. Rev. B 72, 224409 (2005).

10 C. Weber, A. Laeuchli, F. Mila, and Th. Giamarchi, Phys. Rev. B 73, 014519 (2006).

11 M. Renner and W. Brenig, cond-mat/0310244

12 C. Honerkamp, Phys. Rev. B 68, 104510 (2003).

13 N. Bulut, W. Koshibae and S. Maekawa, Phys. Rev. Lett. 95, 037001 (2005).

14 M. C. Refolio, J. M. López Sancho, and J. Rubio, Phys. Rev. B 65, 075114 (2002).

15 S. Moukouri and M. Jarrell, Phys. Rev. Lett. 87 , 167010 (2001).

16 W. Metzner and D. Vollhardt, Phys. Rev. Lett. 62, 324 (1989).

17 E. Müller-Hartmann, Z. Phys. B 74, 507 (1989).
18 A. Georges, G. Kotliar, W. Krauth and M. J. Rozenberg, Rev. Mod. Phys. 68, 13 (1996).

19 Y. Imai and N. Kawakami, Phys. Rev. B 65, 233103 (2002).

20 J. Merino, B. J. Powell, and R. H. McKenzie, Phys. Rev. B 73, 235107 (2006).

21 J. E. Hirsch and R. M. Fye, Phys. Rev. Lett. 56, 2521 (1986).

22 R. M. Fye and J. E. Hirsch, Phys. Rev. B. 38, 433 (1988).

23 M. Jarrell and J. E. Gubernatis, Phys. Rep. 269, 135 (1996).

24 V. M. Galitskii and A. B. Migdal, Zh. Eksp. Teor. Fiz. 34 139 (1958) [Sov. Phys. JETP 7, 96 (1958)]; A. L. Fetter and J. D. Walecka, Quantum Theory of Many-Particle Systems (McGraw-Hill, New York, 1971), Sec. 7.

25 R. T. Scalettar, R. M. Noack, and R. R. P. Singh, Phys. Rev. B 44, 10502 (1991).

26 A. Georges and W. Krauth, Phys. Rev. Lett 69, 1240 (1992).

27 A. Georges and W. Krauth, Phys. Rev. B 48, 7167 (1993).

28 D. Duffy and A. Moreo, Phys. Rev. B 55, 12918 (1997).

${ }^{29}$ For the $2-d$ square lattice, a two-peak structure in $C_{V}(T)$ persists for all interaction strengths. See T. Paiva, C. Huscroft, A. K. McMahan, and R. T. Scalettar, Phys. Rev. B 63, 125116 (2001).

30 M. H. Hettler, A. N. Tahvildar-Zadeh, M. Jarrell, T. Pruschke, and H. R. Krishnamurthy, Phys. Rev. B 58, 7475 (1998).

31 M. H. Hettler, M. Mukherjee, M. Jarrell, and H. R. Krishnamurthy, Phys. Rev. B 61, 12739 (2000).

32 M. Jarrell, Th. Maier, C. Huscroft, and S. Moukouri, Phys. Rev. B 64, 195130 (2001). 